\title{
Tierische Häute - Menschliche Träger. Mensch-Tier-Analogien über die materielle Sachkultur anhand ausgewählter Beispiele im Werk Wolframs von Eschenbach
}

\author{
Dressed in Animal Skin.
}

The relationship between human and animals on the basis of material culture in the work of Wolfram von Eschenbach

Nora Grundtner

\begin{abstract}
By covering the body with animal skin, the fundamental differences between human and animals can change. Wearing the fur of a wild animal, such as a bear, is generally seen as a sign of strength; can wearing luxurious pelts consequently be understood in the same way? The discrepancy between nature and culture is obvious. Pelt, on the one hand, is a sign of a fully established cultural process, but nevertheless it is the skin of a beast which the fictional protagonists of the courtly society wear.

The central question of this article is how the motif of wearing animal skin, and especially pelt, is used in a literary text. Can the apparent difference between roughly tanned animal skin and costly pelts be more than a status symbol?

The article discusses the oscillation between humans and animals by means of wearing fur or pelt in the late medieval tale Aristoteles und Phyllis. Also, to consider human-animal discourse more generally, the ambiguity of the Middle High German term vel will be discussed by the example of passages of pelt wearing figures in the work of Wolfram von Eschenbach.
\end{abstract}

\section{Keywords}

material culture; human animal studies; Middle High German Literature; Wolfram von Eschenbach; Parzival; pelt; fur; skin 
Phillis, diu liehte sunne, gienc
in eine kemenâte hin
und nam ein sîdîn swenzelîn
und leite ez an ir zarten lîp.
daz süeze, minneclîche wîp
het einen pelz darunder,
der was ouch guot besunder;
er gap ir sô blanken schîn
und was gar lûter hermîn.

(Aristoteles und Phyllis, V. 228-236) ${ }^{1}$

In diesem anonym überlieferten Maere beschließt Phyllis den alten Aristoteles, der die Verbindung zwischen Phyllis und Alexander zu unterbinden versucht, zu verführen und ihm dadurch eine Lehre zu erteilen. Dafür zieht sie sich ein Seidenkleid bzw. ein Kleidungsstück mit seidener Schleppe an. Darunter trägt sie einen besonders hell leuchtenden Pelz, Hermelin. Es ist vorstellbar, wenn jedoch mit Blick auf die materielle mittelalterliche Sachkultur unwahrscheinlich, dass sie unter einem Seidengewand ein Pelzhemd oder dergleichen trägt. ${ }^{2}$ Dass Phyllis das Seidenkleid „an ir zarten lîp“ (A. \& P., V. 231) legt, verstärkt die Vermutung, dass sie darunter nichts oder kaum etwas anhat. Darüber hinaus wird durch die Nähe des Glanzes, der Strahlkraft der sonnengleichen Phyllis und dem Schein des hellen bzw. reinen Hermelins eine Verbindung hergestellt. Ziel dieser Nähe ist es nicht allein die Reinheit des Mädchens, sondern die Reize, den Körper und damit die Erotik der Szenerie in den Vordergrund zu stellen. ${ }^{3}$

Dieses mittelhochdeutsche Märe spielt mit der Ähnlichkeit von Mensch und Tier auf diversen Ebenen. So beschreibt der Dichter die schöne Phyllis bewusst mit tierischen Materialien. Der Rezipient wird dabei im Unklaren gelassen, ob von der Haut des Mädchens oder dessen Bekleidung die Rede ist. Der Mensch-Tier-Vergleich ist bereits beim Seidenkleid, swenzelîn, angedeutet. Denn damit wird nicht nur die Schleppe, der untere Teil eines Frauengewandes bezeichnet, sondern auch der Schwanz bzw. Schweif. In späteren Versen kommt es zu einer Anhäufung von Tiervergleichen, besonders von Vögeln: ${ }^{4}$ Darüber hinaus wird Phyllis mit einem Sperber, Papagei und Falken verglichen (vgl. A. \& P., V. 274-281). Der Bereich des Fischfangs (A. \& P., V.543-545) wird wie die

1 Aristoteles und Phyllis. In: Schulz-Grobert, Jürgen (hrsg.) (2006): Kleinere mittelhochdeutsche Verserzählungen. Mittelhochdeutsch/Neuhochdeutsch. Stuttgart: Reclam, S. 28-59 (Nr. II). [im Weiteren auch Sigle A. $\&$ P.]

2 Auch Katrin Kanias Untersuchung zur Kleidung des Mittelalters zeichnet ein anderes Bild. Vgl. Kania, Katrin (2010): Kleidung im Mittelalter. Materialien, Konstruktion, Nähtechnik. Köln [u.a.]: Böhlau.

3 Darüber hinaus wird Phyllis im weiteren Abschnitt noch mit weiteren „Dingen“ verglichen: „ir bein wâren wîzer dan ein slôz,/ und slehter dan ein kerze,/ blanc, ân alle swerze; / diu wurden von dem touwe naz." (Aristoteles und Phyllis, V. 264-267)

4 Das Substantiv mhd. vogelen, vogelfang ist nach Lexer zweideutig und kann „auf den coitus bezogen“" sein. Das Verb mhd. vogelen kann neben dem Fangen von Vögeln auch den Fortpflanzungsakt bezeichnen. Vgl. Wörterbuchnetz - Mittelhochdeutsches Handwörterbuch von Matthias Lexer - vogelen. http://www.woerterbuchnetz.de/Lexer?bookref=3,425,43 (21. 11. 2019). 
Jagd (vgl. A. \& P., V. 309) als Bildgeber für die Beziehung zwischen Aristoteles und Phyllis herangezogen. Dass Aristoteles sich bereiterklärt, durch die Aussicht auf Phyllis Körper, sich als Pferd zur Verfügung zu stellen, „bestiegen“ und „beritten“ zu werden, kann als Fortführung der vorangehende Mensch-Tier-Vergleiche gesehen werden.

Anhand des Beispiels kann gezeigt werden, wie die Tierhaut (am Körper) als Marker einer Auflösung der klaren Grenze zwischen Mensch und Tier fungieren kann. Die Nähe zwischen Mensch und Pelz ist im Maere deutlich und nachvollziehbar. Das Spiel zwischen Tierhaut und Körper erfolgt bei Wolfram von Eschenbach jedoch subtiler, so meine These.

Denn in dem Maere kommt es zu einer Nähe zwischen Mensch und Tier sowohl auf der histoire- als auch auf der discours-Ebene. Auf der Ebene der Geschichte wird Aristoteles zum Tier, in dem er ein Pferd spielt, auf allen Vieren mit einem Sattel auf dem Rücken durch den Garten läuft. Auf der Ebene der Erzählung häufen sich Tiervergleiche und -metaphern. Phyllis wird mit diversen Vögeln und der verführte Aristoteles mit einem auf den Leim - lîmruot (A. \& P., V. 309) - gegangen Vogel verglichen. So rücken sowohl Aristoteles als auch Phyllis zumindest auf Bildebene in die Nähe von Tieren. Die Ankleidung von Phyllis könnte auch als Spiel zwischen histoire- und discours-Ebene gelesen werden. Denn ob Phyllis einen Hermelinpelz trägt oder ob vielmehr von ihrem Körper die Rede ist, das lässt der Erzähler bewusst offen. Bei Wolfram von Eschenbach fehlt in den nachfolgenden Beispielen die Annäherung zwischen Mensch und Tier auf der histoire-Ebene, keine der Figuren verhält sich wie ein Tier. Die Vielzahl an tierischen Metaphern ist ein möglicher Anknüpfungspunkt, die sich aber weniger gut als die erwähnte tierische Materialität fassen lässt, sodass das Tragen von Pelz (auf der Haut) für die Herausarbeitung einer Mensch-Tier-Analogie herangezogen wird.

Nach einem ersten Teil, der sich mit der Etymologie der einschlägigen Begriffe beschäftigt, soll der Mensch-Tier-Diskurs über das Anlegen, Kleiden, Bedecken mit tierischen Häuten einerseits auf Text- aber auch auf Materialebene behandelt werden.

Dafür werden exemplarische Textstellen aus Wolframs Parzival ${ }^{5}$ und Willehalm ${ }^{6}$ herangezogen.

\section{Vel: Etymologie und Material}

Zu Beginn soll auf die begriffliche Mehrdeutigkeit des mhd. Begriffes vel aufmerksam gemacht werden, der nicht nur die tierische, sondern auch die menschliche Haut bezeichnet. ${ }^{7}$

5 Wolfram von Eschenbach (2015): Parzival. Bd. 1 u. 2. Nach der Ausgabe Karl Lachmanns revidiert und kommentiert von Eberhard Nellmann, Übertragen von Dieter Kühn. 4. Aufl. Frankfurt a. M.: Deutscher Klassiker Verlag im Taschenbuch (=Bibliothek des Mittelalters, Bd. 7). [im Weiteren auch Sigle Pz.]

6 Wolfram von Eschenbach (1991): Willehalm. Nach der Handschrift 857 der Stiftsbibliothek St. Gallen. Hrsg., übers. u. komm. v. Joachim Heinzle. Frankfurt a. M.: Deutscher Klassiker Verlag (=Bibliothek des Mittelalters, Bd. 9). [im Weiteren auch Sigle W.]

7 Die zunehmende Differenzierung zwischen Haut (unbehaart) und Fell (behaart) erfolgt erst ab dem 16. Jh. In manchen Sprichwörtern und Redewendungen ist das „Fell“ als Bezeichnung der menschlichen Haut noch erhalten, z. B. Jemandem das Fell über die Ohren ziehen. - vgl. Lloyd, Albert - Lühr, Rosemarie (2007): 
Die fehlende lexikalische Unterscheidung zwischen menschlichen und tierischen Häuten ist nicht allein ein Spezifikum des Mittel- bzw. Althochdeutschen, sondern zeichnet sich auch in anderen Sprachen ab. ${ }^{8}$ So kann mit lat. cutis und pellis die Haut von Menschen und das Fell von Tieren gemeint sein. Cutis und hut werden auf die indogermanische Wurzel $*(s) k e u(\partial)^{9}$ und pellis und vel auf indogermanisch ${ }^{*} p e l^{10}$ zurückgeführt, die beide u. a. die Bedeutung ,bedecken' umfassen.

Ähnlichkeiten zeigen sich ebenfalls auf materieller Ebene. So gibt es zwischen der Haut des Menschen und der Haut von Tieren in Bezug auf die Struktur kaum Unterschiede. Der Mensch unterscheidet sich von anderen Säugetieren in Bezug auf die GröBe des Gehirns, die Dicke der Haare und der Haut. ${ }^{11}$ Dass auch die Haut des Menschen zum großen Teil mit Haaren bedeckt ist, sollte aber nicht außer Acht gelassen werden, sodass der wesentliche Unterschied lediglich in der Dichte der Behaarung liegt. ${ }^{12}$ Das Haar kann also, je nach Dichte, sowohl als Unterscheidungsmerkmal als auch als sichtbares Kennzeichen von Tierähnlichkeit verstanden werden und soll als Anknüpfungspunkt für weitere Überlegungen dienen.

\section{Mensch und Tier}

Der Grundstein für den Mensch-Tier-Diskurs über das Anlegen von Tierhäuten ist, so könnte man argumentieren, bereits in der Genesis vorgezeichnet, denn aus Tierfellen ist die Bekleidung gefertigt, die Gott Adam und Eva gibt, als sie aus dem Paradies vertrieben werden (vgl. 1. Mose 3,21). Das Menschsein, die Bedeckung der Scham, geht, nach dem ersten Bedeckungsversuch mit Blättern, mit der Schlachtung und dem Tragen von Tierhäuten einher. Der Mensch, im Gegensatz zum Tier, bedeckt sich, muss die Dünnheit seiner Haut mit einer weiteren ergänzen. ${ }^{13}$ Das Tier ist dem Menschen dahingehend überlegen, dass es keiner Kleidung bedarf. ${ }^{14}$ Das Anlegen von Fell kann als Menschwerdung Adams und Evas gelesen werden und damit auch als Zeichen, nun

Etymologisches Wörterbuch des Althochdeutschen. Bd. III. fadum-fûstslag. Göttingen: Vandenhoeck \& Ruprecht 2007, S. 122f.

8 „Das griechische zóon kann übrigens, ebenso wie das lateinische animal, sowohl Mensch als auch Tier meinen." Kompatscher, Gabriela [u.a.]: Human-Animal Studies. Eine Einführung für Studierende und Lehrende. Münster/New York: Waxmann 2017, S. 32.

$9 \quad$ Kluge, Friedrich (2011): Etymologisches Wörterbuch der deutschen Sprache. Bearb. v. Elmar Seebold. 25. durchg. u. erw. Aufl. Berlin/Boston: De Gruyter, S. 400.

10 Ebd., S. 286.

11 Vgl. Anzieu, Didier (1991): Das Haut-Ich. Übers. v. Meinhard Korte u. Marie-Hélène Lebourdais-Weiss. Frankfurt a. M.: Suhrkamp, S. 128.

12 Vgl. Karges, Bernadette (2009): Haare. In: Jan Koolman [u.a.] (Hg.): Kaffee, Käse, Karies... Biochemie im Alltag. Weinheim: Wiley-Vch, S. 298-312 (hier S. 298f).

13 Vgl. Benthien, Claudia: Haut. Literaturgeschichte - Körperbilder - Grenzdiskurse. Reinbek bei Hamburg: Rowohlt Taschenbuch 1999, S. 114.

14 Vgl. McCracken, Peggy (2017): In the skin of a beast. Souvereignty an Animality in Medieval France. Chicago/London: The University of Chicago Press, S. 32. 
sterblich zu sein. Andererseits ließe sich das Anlegen von Tierhäuten als Tierähnlichkeit des Sünders interpretieren. ${ }^{15}$

Nach dem Brudermord, dessen Auslöser ein Tieropfer war (vgl. 1. Mos. 4,3-8), erfolgt der erste Betrug mithilfe einer Tierhaut: Rebekka bindet dem haarlosen Jakob Ziegenfelle um die Hände, um dem blinden Vater Isaak vorzuspielen, es sei Esau, damit dieser den zweiten Sohn anstelle des ersten segne (vgl. 1. Mose 27,16).

In der Auseinandersetzung um das Erstgeburtsrecht ist die grundlegende Diskussion über Kultur und Natur bereits vorgezeichnet. Während glatte Haut, gepflegtes Haar als Zeichen von Kultur, Zivilisation (z. B. Jakob, der bei den Zelten bleibt und die Schafe hütet) gilt, ist das struppige Haar, Körperbehaarung etwas außerhalb der Zivilisation Stehendes (z. B. der Jäger Esau) ${ }^{16}$. Starke Körperbehaarung sei, so Konrad von Megenberg (14. Jahrhundert), ${ }^{17}$ ein sichtbares Zeichen großer Körperhitze. Je behaarter ein Mensch und überraschenderweise auch ein Tier, desto unkeuscher ist es und Konrad von Megenberg beruft sich in seinen Ausführungen auf Aristoteles: „Ariftotiles fpricht, daz ein iegleich tier, daz vil hars hab, vnd ein iegleich menfch vnchäufcher fei dann ain anders, vnd auch ein iegleich vogel, der mer federe hat denn ein ander." (Konrad von Megenberg: Buch der Natur, I.3, V. 23-27) ${ }^{18}$

Tierähnlichkeit (hier: Behaarung) ist also nicht allein ein äußeres Zeichen, sondern Ausdruck eines lasterhafteren, unmäßigeren Inneren. ${ }^{19}$ Haarlosigkeit als antikes Ideal lässt sich in Ovids Liebeslektionen an die Frauen (ars amatoria) ${ }^{20}$ nachlesen: „Beinah hätt‘ ich gemahnt: Nicht komme der trotzige Bock euch unter die Achseln; nicht sei rau von den Härchen das Bein!“21 (Aa., V. 193f).

Der zivilisierte Mann rasiert sich, stutzt und pflegt seinen Bart. ${ }^{22}$ Dass sich die zivilisierte Frau enthaart, sich aber mit dem Pelz von Tieren schmückt, ${ }^{23}$ bietet Raum für weitere Spekulationen und führt zu der Frage, ob sich die Unterscheidung zwischen Kultur

15 Vgl. ebd., S. 16f.

16 „Esau wurde ein Mann, der sich auf die Jagd verstand, ein Mann des freien Feldes. Jakob war ein Mann ohne Fehl und blieb bei den Zelten." (1. Mose 25,27).

17 Vgl. Pfeiffer, Franz (1962): Einleitung. In: Konrad von Megenberg. Das Buch der Natur. Hrsg. v. Franz Pfeiffer. Hildesheim: Georg Olms Verlagsbuchhandlung, S. V-LXII (hier S. IX).

18 Konrad von Megenberg (2011): Buch der Natur, Bd. I. Kritischer Text nach den Handschriften. Hrsg. v. Robert Luff u. Georg Steer. Berlin/Boston: De Gruyter. https://www.degruyter.com/viewbooktoc/product/24911 (25. 4. 2019). [im Weiteren auch Sigle BdN.]

19 Vgl. Hintz, Ernst Ralf (1999): Der Wilde Mann - ein Mythos vom Andersartigen. In: Ulrich Müller, Werner Wunderlich (Hg.): Dämonen Monster Fabelwesen. St. Gallen: UVK (=Mittelalter: Mythen, Bd. 2), S. 619-626. (hier S. 620).

20 Ovidius Naso, Publius (2011): Liebeskunst. lateinisch-deutsch. Liber secundus. Hrsg. v. Niklas Holzberg. 5. überarb. Aufl. Berlin: Akad.-Verl. https://www.degruyter.com/viewbooktoc/product/227425 (17.04.2019).

[im Weiteren auch Sigle Aa.]

21 quam paene admonui, ne trux caper iret in alas/ neve forent duris aspera crura pilis! (Aa., V. 193f).

22 nec male deformet rigidos tonsura capillos; sit coma, sit trita barba resecta manu. Aus: Ovidius Naso, $\mathrm{Pu}-$ blius (2011): Liebeskunst. lateinisch-deutsch. Liber primus. Hrsg. v. Niklas Holzberg. 5. überarb. Aufl. Berlin: Akad.-Verl. https://www.degruyter.com/viewbooktoc/product/227425 (17.04.2019), V. 517f.

23 Betancourt Nuñez, Gabriele (2007): „Nimm dich in Acht vor ihren schönen Haaren“. In: Herlinde Koelbl: Haare. Publikation anlässlich der Ausstellung. Ostfildern: Hatje Cantz, S. 156-165 (hier S. 160). 
und Natur, Haarlosigkeit und Behaartheit auch in Bezug auf die Tierhaut bzw. Tierhaar tragenden Menschen anwenden lässt.

Die Nähe zwischen Mensch und Tier wird, so meine These, über die materielle Kultur vorgenommen. Anhand von Wolframs von Eschenbach Oeuvre soll gezeigt werden, wie einerseits die begriffliche Mehrdeutigkeit von mhd. vel, anderseits auch die ähnliche Beschaffenheit von Haut und Fell/Pelz Anknüpfungspunkt für mögliche Lesarten der Texte sein können.

\section{Fell und Pelz im Werk Wolframs von Eschenbach}

Der Verdacht, Begriffe wie Haut, Haar, Fell, Pelz würden bei Wolfram stärker im Vordergrund stehen, wurde durch eine quantitative Analyse exemplarischer Texte von Dichtern des 12. und 13. Jahrhunderts bestärkt. Die $\mathrm{MHDBDB}^{24}$ wurde nach einschlägigen Ausdrücken rund um das Thema Haut und Pelz befragt, wie hût, vel, bellîz, balc, hermîn, zobel. ${ }^{25}$

\begin{tabular}{|r|c|c|c|c|c|}
\hline Begriffe & Heinrich v. Veldeke & $\begin{array}{l}\text { Hartmann von } \\
\text { Aue }\end{array}$ & Nibelungenlied & $\begin{array}{l}\text { Wolfram } \\
\text { v. Eschenbach }\end{array}$ & $\begin{array}{l}\text { Gottfried } \\
\text { v. Straßburg }\end{array}$ \\
\hline hût & 10 & 4 & $5,5^{26}$ & 14 & 1 \\
\hline vel & 1 & 5 & - & 52 & 1 \\
\hline bellîz & 1 & 1 & - & 3 & - \\
\hline balc & - & - & - & 7 & - \\
\hline hermîn & 5 & 6 & 2 & 12 & 3 \\
\hline zobel & 4 & 8 & 3 & 15 & 4 \\
\hline
\end{tabular}

Tab. 1: Begriffssuche im Werk einschlägiger Dichter des 12. und 13. Jahrhunderts

Der Anschein, es könne sich um ein Wolframsches Spezifikum handeln, wird durch die Anzahl der Begriffe, insbesondere von vel, bestätigt. Jedoch handelt es sich beim Euvre Wolframs auch um das umfangreichste. Daher muss die Anzahl der Begriffe im Verhältnis zur Wortanzahl betrachtet werden.

24 Mittelhochdeutsche Begriffsdatenbank (MHDBDB). Universität Salzburg. Koordination: Katharina Zeppezauer-Wachauer. Technische Leitung: Peter Hinkelmanns, Daniel Schlager. 1992-2018. http://www.mhdbdb.sbg.ac.at/ (November, Dezember 2018; April 2019).

25 Permint wird im Parzival dreimal, in der Eneide zweimal und im Nibelungenlied einmal erwähnt.

26 Die Anzahl der Begriffe (4-9) in den unterschiedlichen Handschriften wurden addiert und durch die Anzahl der einbezogenen Handschriften dividiert. 


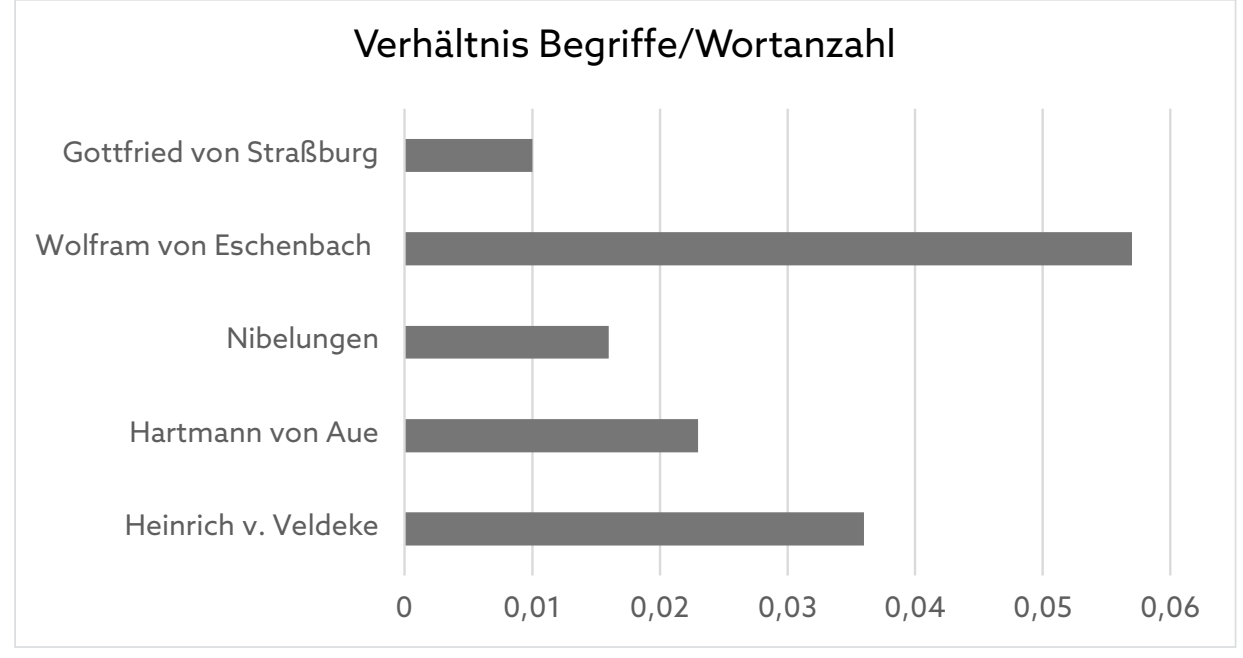

Tab. 2: Begriffe im Verhältnis zur Wortanzahl

In der Grafik anschaulich dargestellt, wird die Annahme, Wolfram verwende Begriffe rund um den Themenkomplex ,Fell‘ und ,Haut` häufiger, bestätigt.

\section{hût und vel}

Die 52 Erwähnungen des Lexems vel in Wolframs Werk lassen aufhorchen. Dass der Ausdruck vel bei Wolfram besonders markiert ist, zeigt sich darüber hinaus in der Lyrik. Denn während beispielsweise hût, etwa bei Walther, durchaus erwähnt wird, finden sich vor Wolfram keine Belege in der Lieddichtung.

In der dritten Strophe von Wolframs Tagelied, Den morgenblic bî wahtceres sange erkôs, heißt es:

Der trûric man nam urloup balde alsus

ir liehten vel diu slehten

kômen nâher. sus der tac erschein. ${ }^{27}$

Begriffe wie hermîn, zobel in der Lieddichtung sind ebenfalls rar, da fällt Neidharts Spiel zwischen Pelz und Haut in einem seiner Lieder auf:

Wer ich aber ein decklach hermlein

einer seuberlichen magt

oder einer frawen,

27 Wolfram von Eschenbach: Den morgenblic bî wahteres sange erkôs. In: Deutsche Lyrik des frühen und hohen Mittelalters (2006). Hrsg. u. komm. v. Ingrid Kasten. Übersetzungen von Margherita Kuhn. Frankfurt a. Main (=Deutscher Klassiker Verlag im Taschenbuch, Bd. 6), S. 534-536. 
als ein mantel, der da wär von palczingin,

den die frawen gern tragen,

wenn si ritter schawent.

(Neidhart, das seiden risel c 95, XI, 1-6) (28 $^{2}$

Dieser Wunsch ist der dritte, den das männliche Ich in diesem Lied zum Ausdruck bringt. Zuerst wünscht er sich ein „seyden reyslein“ (N. c 95 IX,1) zu sein, damit er die Wangen der Liebsten berühren kann, danach wäre er gern ein „gurtell den die lieb umb trug“ (N. c 95 X,1). So ist der Wunsch, die Liebste vollkommen zu bedecken, lediglich die Darstellung der letzten Phase einer Annäherung, nach dem Streicheln der Wange und dem Umfassen der (Taille der) Liebsten.

Der Mantel einer Frau zu sein, mit diesem Bild spielt die Gawan-Fährmann-Episode im Parzival (Pz. 552-555). Gawan erhält vor dem Schlafengehen den Hermelinmantel Benes, der ihm als Decke dient: „man leit ein wanküssen dar, unt der meide mantel einen, härmîn niwe reinen. “29 (Pz. 552, 20-23) Und als Gawan zu Bett geht, heißt es: „der meide mantel übervienc/ in: daz was sîn decke." (Pz. 553,21f)

Die offensichtlich erotische Anspielung, wird durch die Reaktion des Vaters auf Benes Tränen am nächsten Tag verdeutlicht. (Pz. 552,22) Das Weiß des Hermelins korrespondiert mit der hellen Haut - zumindest der Hände ${ }^{30}$ (vgl. Pz. 551,7) - der meide wol gevar (Pz. 551,11). ${ }^{31}$

Das Spiel zwischen dem Träger, der Trägerin eines Pelzes und dem darunterliegenden Körper liegt sowohl am Beispiel Neidharts als auch an Benes Mantel nahe.

Deutlich subtiler, und in der Forschungsliteratur weit weniger beachtet, erfolgt die Nähe zwischen Körper und Pelzdecken in weiteren Szenen in Wolframs Parzival, die mit der gleichen Bedeutung (,bedecken') der unterschiedlichen indogermanischen Wurzeln von hut und vel spielen. ${ }^{32}$

28 Neidhart-Lieder. Texte und Melodien sämtlicher Handschriften und Drucke. Bd. 2 (2011). Hrsg. v. Ingrid Bennewitz [u.a.]. Berlin/Boston: De Gruyter. https://www.degruyter.com/viewbooktoc/product/62205 (23. 4. 2019). [im Weiteren auch Sigle N.]

29 Auch durch das Spiel von Küssen und Kissen bzw. hier zusätzlich zwischen Kopfkissen und Wangenkuss: wange-küsse, fließt eine erotische Komponente ein.

30 Für manche antike und mittelalterliche Philosophen ist genau die Hand, besonders beim Essen, das, was den Menschen vom Tier unterscheidet; vgl. McCracken [Anm. 14], S. 82f. Darüber hinaus kann das Weiß des Mantels sicherlich auch als Anspielung auf Benes Jungfräulichkeit, siehe die Reaktion des Vaters, gelesen werden.

31 Die erotischen Anspielungen werden zusätzlich durch Benes wiederholte Hilfestellungen beim Rüstungsablegen verstärkt (vgl. Pz. 549,15; 621,27). Beim Rückweg kommt Gawan wieder beim Fährmann vorbei und er trägt beim zweiten Aufenthalt Benes Mantel über dem kursît (vgl. Pz. 621,28-622,3).

32 indogermanisch *(s)keu(ə) und indogermanisch*pel. Kluge: Etymologisches Wörterbuch deutscher Sprache, S. 400; 286. 


\section{Scham - Schamhaftigkeit - Unverschämtheit}

Parzival erblickt die schlafende Jeschute, die nur bis zur Hüfte mit der Pelzdecke zugedeckt ist (vgl. Pz. 130,17). Die halb mit einem Zobelpelz zugedeckte Schlafende lässt unweigerlich an Darstellungen der Venus Pudica denken.

Venus selber ja beugt, sooft die Hüllen sie ablegt, halb sich zurück, und die Scham deckt mit der Linken sie zu. Überall und öffentlich paaren sich Tiere; ein Mädchen, wenn es so etwas erblickt, wendet doch ab das Gesicht. Tür und Kammer gehören zu unserm verborgenen Treiben, und der Schambereich bleibt von der Decke verhüllt; zwar nicht Finsternis, doch ein gewisses nebliges Dunkel suchen wir, etwas, das weniger hell ist als das Licht.. ${ }^{33}$ (Aa., V. 613-620)

Jeschute, so könnte man wie das Martina Feichtenschlager in ihrer Arbeit darlegt, formulieren, wird über den Umweg der Kleidung, hier: Bedeckung, entblößt. ${ }^{34}$ Die dunkle Tierhaut verhüllt den ebenfalls (dunkel?) behaarten Schambereich. ${ }^{35}$

Die Decke, die eventuell darunter stattfindendes Treiben verhüllt, wird dem schlafenden Königspaar Artus und Ginover durch Segramors entzogen. (Pz. 285,16f)

In direkter Verbindung mit der Haut steht das „declachen zobelîn“ bei Gahmuret und Belakane, wird doch die dunkle Haut der Königin in die Nähe des dunklen Zobels gestellt. (Pz. 44,20) Die Verbindung von Belakanes Haut und der Farbe des Zobels zeigt sich in der Beschreibung Gahmurets Mantel: „ein zobel dâ vor gap swarzen schîn“ (Pz. 63,24). Ähnlich wird die Dunkelheit Belakanes beschrieben, wenn es heißt: „nâch swarzer varwe was ir schîn“. (Pz. 24,11)

Die Verbindung aus „schîn“ und „schwarz“ erfolgt nur in der Beschreibung von Belakanes Haut und Gahmurets Zobel.

ir kiusche was ein reiner touf,

und ouch der regen der sie begôz,

der wâc der von ir ougen flôz

ûf ir zobel und an ir brust. (Pz. 28, 14-17)

33 ipsa Venus pubem, quotiens velamina ponit,/ protegitur laeva semireducta manu,/ in medio passimque coit pecus; hoc quoque viso/ avertit vultus nempe puella suos./ conveniunt thalami furtis et ianua nostris,/ parsque sub iniecta veste pudenda latet,/ et, si non tenebras, at quiddam nubis opacae/ quaerimus atque aliquid luce patente minus.

34 Feichtenschlager, Martina (2016): Entblößung und Verhüllung. Inszenierungen weiblicher Fragilität und Verletzbarkeit in der mittelalterlichen Literatur. Göttingen: V \& R, S. 11f.

35 Die Jeschute-Episode erinnert an Sigunes Hemdgeschenk an Schoynatulander in Wolframs Titurel. Das Kleidungsstück wird dabei folgendermaßen beschrieben: „Im gap dar diu künegin ir hemde/ blanc sîdîn, als ez ir blenke ruorte./ ez ruorte douch etwas brûnes an ir huf. Den poneiz vor Baldac erz fuorte." (Titurel 85,24, im Weiteren auch T.) Das Braune, das das Hemd Sigunes berührt hat, lässt sich unschwer als eine Anspielung auf Sigunes Schambehaarung lesen. Vgl. Brackert, Helmut; Fuchs-Jolie, Stephan: Stellenkommentar. In: Wolfram von Eschenbach (2003): Titurel. Hrsg., übers. u. komm. v. dies. Berlin/New York: Walter de Gruyter, S. $205 f$. 
Die Nähe zu Belakanes Haut wird an dieser Stelle nicht allein über den ein Körperteil bezeichnenden Begriff „brust“ sondern zusätzlich über den Umweg einer tierischen Haut, die zumindest eine gemeinsame Eigenschaft besitzt: dunkler Farbe zu sein, hergestellt. Denn der Zobel ist die kleinste Art der Echten Marder, ${ }^{36}$ die ein dunkles - je dunkler desto kostbarer - Fellkleid besitzt. ${ }^{37}$ Das zusätzliche Benetzen der (tierischen?) Haut mit Tränen, ${ }^{38}$ einer (christlichen) Taufe ähnlich, lässt an die besonders begehrten Zobelpelze erinnern, die einen seidigen Schimmer besitzen, der als „Wasser“ bezeichnet wird. ${ }^{39}$ Dass auf Belakanes Körper durch das Erwähnen des Fells angespielt wird, liegt u. a. auch deshalb nahe, da Belakanes Kleidung an keiner Stelle im Parzival erwähnt und das Material Zobel davor nur in Bezug auf Gahmurets Schild erwähnt wird (vgl. Pz. 18,6f). Es ist das einzige („textile“) Material, mit dem Belakane in Verbindung gebracht wird. Darüber hinaus wird durch das Possessivpronomen ir eine Nähe zu Belakane selbst aufgebaut. Das Material Zobel wird an keiner Stelle als pars pro toto für ein Kleidungsstück erwähnt, pelliz hingegen schon (vgl. Pz. 231,5). Es ist stets die Rede von Zobel als Material für Besatz (vgl. Pz. 168,13f), Rüstungsschmuck (vgl. Pz. 575,26f), Decken (Pz. 285,16), Schilder (vgl. Pz, 101,7f), Wimpel (vgl. Pz. 673,14f) und dergleichen. Wie die weiße Haut der Phyllis mit Hermelin verglichen wird (vgl. A. u. P., V. 236), scheint hier die menschliche Haut so dunkel wie Zobel zu sein. Der Vorwurf Konrads, behaarte Häute seien ein Zeichen von Unkeuschheit (vgl. BdN., I.3, V. 23-27), wie sie in dem Changieren zwischen Pelz und Körper bei Phyllis angedeutet und über den weiteren Verlauf der Erzählung mehr als deutlich wird, klingt auch hier, im indirekten Mensch-Tier-Vergleich über die Erwähnung des Pelztieres Zobel an. Der dunkle Zobelpelz und die dunkle Haut Belakanes stehen in einem Ähnlichkeitsverhältnis zueinander, sodass der verhüllende Pelz auf den darunter liegenden Körper der schönen Belakane rekurriert und diesen somit indirekt entblößt. Diese Anspielung eröffnet eine erotische Ebene, die den Begriff der ,Wildheit' und des außerhalb der Zivilisation Stehende anspricht und einen erweiterten Blick auf die materielle Pelzkultur in der Literatur ermöglicht. Inwieweit in dem Material das ursprüngliche Tier ersichtlich ist bzw. dem Träger wie Rezipienten bewusst ist, dass es sich um Tierhaut handelt, muss eigens diskutiert werden. Jedoch bezeichnet der Begriff zobel ja nicht nur die Tierhaut, den Pelz, sondern auch das Tier.

Dass in den exemplarischen Analysen bisher lediglich Stellen aus dem Parzival herangezogen wurden, rührt daher, dass ein Großteil der Haut- und Fellbegriffe vorwiegend und z. T. ausschließlich im Parzival erwähnt sind. Allerdings finden sich auch in den anderen Werken Wolframs Mensch-Tier-Analogien, die aber nur zum Teil über die Begrifflichkeiten der material culture hergestellt werden.

36 Vgl. Tuma, Alexander (1951): Pelzlexikon: Rauhwarenhandel - Zyperkatze. Wien: Alexander Tuma (=Pelz- und Rauhwarenkunde, Bd. 21), S. $290 f$.

37 Vgl. ebd., S. 293.

38 Ebenfalls tränenfeucht ist die Kleidung vom Gefolge Gahmurets Bruder: „ir wât wart von den ougen naz“ (Pz. 99,3), als sie zu Gahmuret eilen. Hier ist die Bekleidung/Materialität nicht markiert, die tränennassen Kleider zeigen die Trauer und das Berührtsein des Gefolges an (vgl. Pz. 99,3-6).

39 Vgl. Tuma [Anm. 36], S. 293. 


\title{
Jäger und Gejagter
}

Die bereits in Gahmurets Wappen (und zwar genauer: in der Heraldik) vorweggenommene Nähe zwischen (tierischer) Haut und Kampf wird im Willehalm [im Weiteren auch W.] aber auch im Titurel fortgeführt.

\author{
si pruoften sein gereite, \\ daz ûf dem wundem orse lac, \\ und eines sites, des er pflac, \\ daz er ein klein belzelîn \\ (daz selbe was lieht hermîn) \\ an zôch, dar ob er wâpen truoc \\ (des belzelîns ein gêre sluoc \\ hinden übern satelbogen); \\ und dô Puzzât vür unbetrogen \\ sô eben zogt ûf sîner slâ, \\ des bekanden in die heinden dâ. (W. 84,20-30)
}

Willehalm wird zum gejagten Tier, wenn die Hermelinbekleidung, die er unter der heidnischen Rüstung trägt, hervorblitzt und den Feinden seine wahre Herkunft verrät. (W. 84,25; 85,13) Hier stellt sich die Frage, weshalb das Hermelinfell diese Signalfunktion besitzt. Das Hermelin ist ein Tier, das im Norden Europas und Sibirien ${ }^{40}$ beheimatet ist und dessen weißes Winterfell als besonders kostbar gilt. ${ }^{41}$ Der Pelz ist also ein auffallendes Zeichen für Willehalms europäische (hier: französische) Herkunft. Neben dem Pelz ist sein verletztes Pferd, das Willehalm losgebunden hat, ihm aber trotzdem folgt (vgl. W. 82,9-14), für die Heiden ein Indiz, dass ein Christ auf dessen Rücken sitzt (vgl. W. 85,10). So ist es einerseits das Tier, das Willehalm als Kleidung trägt, andererseits die Beziehung zwischen Tier und Reiter, die die Sarazenen stutzig macht. ${ }^{42}$ Während er die Sarazenen in seinem Aufzug nicht zu täuschen vermag, gelingt ihm das bei Gyburc, die aufgrund seiner Aufmachung nicht glauben kann, er sei Willehalm (vgl. W. 89-92).

Inwiefern das Tragen von Pelz unter dem Harnisch verbreitet war, kann schwer beurteilt werden, es scheint jedoch auffällig und möglicherweise eine Eigenart Willehalms zu sein, die aber abgesehen von diesen beiden Stellen im Versroman keine Rolle mehr spielt. Auch als Willehalm aus Trauer über die Trennung von Gyburc seine ungemütliche Rüstung anbehalten und auf teure Seidenkleider verzichten möchte, wird nicht erwähnt, was Willehalm unter seiner Rüstung trägt (vgl. W. 174,3-13). Im Katalog zu „Kleidung im Mittelalter“ Katrin Kanias findet sich keine Unterkleidung aus Pelz, was aber aufgrund der geringen Anzahl historischer Fundstücke nicht verwundern muss.

40 Hermeline finden sich auch in Nordamerika.

41 Vgl. Tuma, Alexander (1949): Pelzlexikon. Fachliteratur - Kaninfell. Wien: Verlag Alexander Tuma 1949 (=Pelz- und Rauhwarenkunde, Bd.18), S. 101.

42 Auch das Sattelzeug des Pferdes weckt das Misstrauen (vgl. W. 84,20f). 
Generell scheint es aber für Oberbekleidung üblicher gewesen zu sein, den Pelz nach innen, also als Futter, und nicht nach außen, vom Besatz abgesehen, gewendet zu tragen. ${ }^{43}$

Der Bezug auf Willehalms Haut ist hier weniger vordergründig, da ja sein Pelzkleid länger als die Rüstung zu sein scheint und über den Sattel hängt (vgl. W. 84,26). Jedoch erfolgt die Nähe zwischen dem Tiermaterial und Willehalm über die Jagdmetaphorik. Willehalm wird zur besonderen (Jagd-)Trophäe, denn er hat den Sarazenen durch seinen tapferen Kampf große Verluste bereitet (vgl. W. 85,1-9).

Die Verschmelzung zwischen Jäger und Gejagtem erfolgt auch in Titurel (im Weiteren auch T). So gleicht Schionatulander, dessen Beine bei der Jagd nach dem Hund ganz zerkratzt werden, einem erlegten Tier:

Im wurden diu blôzen bein ganz zerkratzet

die sînen blanken füeze an dem loufe ouch

man kôz in, baz danne daz erschozen tier, wunden.

er hiez si twahen, ê er kœme von den brâmen.

von stuften ein teil wunden nâmen.

underz gezelt. [...] (T. 166,1-4)

In diesem Textbeispiel wird die verletzte Haut Schionatulanders direkt, ohne den Umweg einer getragenen Tierhaut, mit einem gejagten Tier in Beziehung gesetzt.

\section{Resümee}

Anhand der angeführten Textpassagen sollte eine Lektüre der materiellen Sachkultur von Haut und Pelz als ein möglicher Zugang zum Text vorgestellt werden.

Die grundlegende Frage war dabei, ob die Ähnlichkeit der Materialität von menschlicher und tierischer Haut und die Unschärfe des mittelhochdeutschen Begriffs vel als Basis einer weiter gefassten Analyse des Mensch-Tier-Diskurses in Wolframs Werk herangezogen werden und inwieweit dies als ein Spiel zwischen tierischer Haut und menschlichem Träger aufgefasst werden kann.

Während die Dichte der Behaarung menschlicher Akteure als ein Anzeichen außerhalb der Gesellschaft zu stehen, gelesen werden kann, ist die Einordnung Tierhaut tragender Figuren komplexer. In beiden Fällen können die Figuren in die Nähe der Tierwelt rücken bzw. kann die strikte Trennung zwischen Tierischem und Menschlichem verschwimmen. Doch während die dicht behaarte/ungepflegte Haut oder das Anlegen unhöfischer Felle ein auffälliges Zeichen von Askese, Armut oder Wildheit ist, gilt das Tragen von Pelz als ein Signal für Reichtum. Dass Pelz als mehr als ein luxuriöses Zeichen fungieren kann, konnte exemplarisch anhand dem Maere Aristoteles und Phyllis gezeigt werden. Der Vergleich zwischen Mensch und Tier vollzieht sich in dieser Erzählung sowohl auf der Ebene der Handlung, als auch auf Ebene der Erzählung, sodass das Anlegen von Pelz und das damit verbundene Rekurrieren auf Phyllis‘ Körper nur eines von mehreren Mensch-Tier-Markern ist.

43 Vgl. Kania [Anm. 2], S. 410; 424; 426; 431. 
Dezenter erfolgen die Mensch-Tier-Vergleiche im Werk Wolframs von Eschenbach, die Figuren werden nicht zum Tier oder verhalten sich nicht bewusst tierisch, wie der auf allen Vieren kriechende und Pferd spielende Aristoteles. Die Figuren in den Werken Wolframs von Eschenbach werden als Pelzträger charakterisiert und dieses Anlegen von Tierfell konnte anhand exemplarischer Analysen als ein Ausgangs- und Anknüpfungspunkt einer weiter gefassten Mensch-Tier-Analogie skizziert werden.

Durch das Tragen von luxuriösen Pelzen, die trotzdem aus Tieren gefertigt sind, verstärkt sich die Spannung zwischen Natur und Kultur. Denn diese sind einerseits Ausdruck größten Luxus` und das Ergebnis eines aufwendigen und ausgefeilten Kulturprozesses, was aber nichts daran ändert, dass es sich um Tierhaut handelt, mit der die (höfischen) Akteure gekleidet und geschmückt dargestellt werden. Das Spiel zwischen Jäger und Gejagtem, die Metaphorik von Kampf schwingt dabei in all den erwähnten Passagen mit und ist besonders in Bezug auf die Erotik, den Liebeskampf, präsent. Das Changieren zwischen Mensch und Tier über die Materialität von Fell und Pelz kann anhand von Beispielen in Wolframs Werk nachvollzogen werden.

\section{Literaturverzeichnis}

\section{Primärliteratur}

Aristoteles und Phyllis. In: Schulz-Grobert, Jürgen (hrsg.) (2006): Kleinere mittelhochdeutsche Verserzählungen. Mittelhochdeutsch/Neuhochdeutsch. Stuttgart: Reclam, S. 28-59, Nr. II (=Reclams Universal-Bibliothek 18431). (Sigle A. \& P.)

Die Bibel. Einheitsübersetzung der Heiligen Schrift (2016). Vollständig durchges. und überarb. Ausgabe. Hrsg. v. Katholischen Bibelanstalt. Stuttgart: kbw Bibelwerk. https://www.bibleserver.com/ (23. 4. 2019).

Deutsche Lyrik des frühen und hohen Mittelalters (2006). Hrsg. u. komm. v. Ingrid Kasten. Übersetzungen von Margherita Kuhn. Frankfurt a. Main (=Deutscher Klassiker Verlag im Taschenbuch, Bd. 6).

Jacobus de Voragine (2007): Legenda Aurea. Übers. v. Richard Benz. 15. Aufl. Gütersloh: Gütersloher Verlagshaus.

Konrad von Megenberg (2011): Buch der Natur, Bd. I. Kritischer Text nach den Handschriften. Hrsg. v. Robert Luff u. Georg Steer. Berlin/Boston: De Gruyter. https://www.degruyter.com/ viewbooktoc/product/24911 (25. 4. 2019). (Sigle BdN.)

Neidhart-Lieder. Texte und Melodien sämtlicher Handschriften und Drucke. Bd. 2 (2011). Hrsg. v. Ingrid Bennewitz [u.a.]. Berlin/Boston: De Gruyter. https://www.degruyter.com/viewbooktoc/product/62205 (23. 4. 2019). (Sigle N.)

Ovidius Naso, Publius (2011): Liebeskunst. lateinisch-deutsch. Liber primus. Hrsg. v. Niklas Holzberg. 5. überarb. Aufl. Berlin: Akad.-Verl. https://www.degruyter.com/viewbooktoc/product/227425 (17.04.2019).

Ovidius Naso, Publius (2011): Liebeskunst. lateinisch-deutsch. Liber secundus. Hrsg. v. Niklas Holzberg. 5. überarb. Aufl. Berlin: Akad.-Verl. https://www.degruyter.com/viewbooktoc/product/227425 (17.04.2019). (Sigle Aa.)

Wolfram von Eschenbach (2015): Parzival. Bd. 1 u. 2. Nach der Ausgabe Karl Lachmanns revidiert 
und kommentiert von Eberhard Nellmann, Übertragen von Dieter Kühn. 4. Aufl. Frankfurt a. M.: Deutscher Klassiker Verlag im Taschenbuch (=Bibliothek des Mittelalters, Bd. 7). (Sigle Pz.)

Wolfram von Eschenbach (2003): Titurel. Hrsg., übers. u. komm. v. Helmut Brackert u. Stephan Fuchs-Jolie. Berlin/New York: Walter de Gruyter. (Sigle T.)

Wolfram von Eschenbach (1991): Willehalm. Nach der Handschrift 857 der Stiftsbibliothek St. Gallen. Hrsg., übers. u. komm. v. Joachim Heinzle. Frankfurt a. M.: Deutscher Klassiker Verlag (=Bibliothek des Mittelalters, Bd. 9). (Sigle W.)

\section{Wörterbücher}

Kluge, Friedrich (2011): Etymologisches Wörterbuch der deutschen Sprache. Bearb. v. Elmar Seebold. 25. durchg. u. erw. Aufl. Berlin/Boston: De Gruyter.

Lloyd, Albert; Lühr, Rosemarie (2007): Etymologisches Wörterbuch des Althochdeutschen. Bd. III. fadum-fûstslag. Göttingen: Vandenhoeck \& Ruprecht 2007.

Wörterbuchnetz - Mittelhochdeutsches Handwörterbuch von Matthias Lexer - vogelen. http:// www.woerterbuchnetz.de/Lexer?bookref=3,425,43 (21. 11. 2019).

\section{Sekundärliteratur}

Anzieu, Didier (1991): Das Haut-Ich. Übers. v. Meinhard Korte u. Marie-Hélène Lebourdais-Weiss. Frankfurt a. M.: Suhrkamp.

Benthien, Claudia (1999): Haut. Literaturgeschichte - Körperbilder - Grenzdiskurse. Reinbek bei Hamburg: Rowohlt Taschenbuch.

Betancourt Nuñez, Gabriele (2007): „Nimm dich in Acht vor ihren schönen Haaren“. In: Herlinde Koelbl: Haare. Publikation anlässlich der Ausstellung. Ostfildern: Hatje Cantz, S. 156-165.

Brackert, Helmut; Fuchs-Jolie, Stephan: Stellenkommentar. In: Wolfram von Eschenbach (2003): Titurel. Hrsg., übers. u. komm. v. dies. Berlin/New York: Walter de Gruyter, S. 143-277.

Feichtenschlager, Martina (2016): Entblößung und Verhüllung. Inszenierungen weiblicher Fragilität und Verletzbarkeit in der mittelalterlichen Literatur. Göttingen: V \& R.

Hintz, Ernst Ralf (1999): Der Wilde Mann - ein Mythos vom Andersartigen. In: Ulrich Müller, Werner Wunderlich (Hg.): Dämonen Monster Fabelwesen. St. Gallen: UVK (=Mittelalter: Mythen, Bd. 2), S. 619-626.

Kania, Katrin (2010): Kleidung im Mittelalter. Materialien, Konstruktion, Nähtechnik. Köln [u.a.]: Böhlau.

Karges, Bernadette (2009): Haare. In: Jan Koolman [u.a.] (Hg.): Kaffee, Käse, Karies... Biochemie im Alltag. Weinheim: Wiley-Vch, S. 298-312.

Kompatscher, Gabriela [u.a.] (2017): Human-Animal Studies. Eine Einführung für Studierende und Lehrende. Münster/New York: Waxmann.

McCracken, Peggy (2017): In the skin of a beast. Souvereignty an Animality in Medieval France. Chicago/London: The University of Chicago Press.

Pfeiffer, Franz (1962): Einleitung. In: Konrad von Megenberg. Das Buch der Natur. Hrsg. v. Franz Pfeiffer. Hildesheim: Georg Olms Verlagsbuchhandlung, S. V-LXII.

Tuma, Alexander (1949): Pelzlexikon. Fachliteratur - Kaninfell. Wien: Verlag Alexander Tuma (=Pelz- und Rauhwarenkunde, Bd. 18). 
Tuma, Alexander (1951): Pelzlexikon: Rauhwarenhandel - Zyperkatze. Wien: Alexander Tuma (=Pelz- und Rauhwarenkunde, Bd. 21).

Walter, Henrike (2008): Flechten, Fell und Bärte. Genderspezifische Aspekte der Haarsymbolik im Märchen. In: Birgit Haas (Hg.): Haare zwischen Fiktion und Realität. Interdisziplinäre Untersuchungen zur Wahrnehmung der Haare. Berlin: LIT (Kulturwissenschaft, Bd. 17), S. 341-362.

\section{Datenbank}

Mittelhochdeutsche Begriffsdatenbank (MHDBDB). Universität Salzburg. Koordination: Katharina Zeppezauer-Wachauer. Technische Leitung: Peter Hinkelmanns, Daniel Schlager. 19922018. http://www.mhdbdb.sbg.ac.at/ (November, Dezember 2018; April 2019).

MMag. Nora Grundtner / nora.grundtner@sbg.ac.at

Paris Lodron Universität Salzburg, Kultur- und Gesellschaftswissenschaftliche Fakultät, Fachbereich Germanistik

Erzabt-Klotzstr. 1, 5020 Salzburg, AT 
